\title{
Prevalence of Acute Coronary Syndrome in Patients Suspected for Pulmonary Embolism or Acute Aortic Syndrome: Rationale for the Triple Rule-Out Concept
}

\author{
Saad Al Qahtani ${ }^{\mathrm{a}, \mathrm{d}}$, Ahmed Y. Kandeel ${ }^{\mathrm{a}}$, Stephane Breault ${ }^{\mathrm{b}}$, Anne-Marie Jouannic ${ }^{\mathrm{b}, \mathrm{c}}$, Salah D. Qanadli ${ }^{\mathrm{b}, \mathrm{c}}$
}

\begin{abstract}
Background: The aims of the study were to evaluate the prevalence of acute coronary syndrome (ACS) among patients presenting with atypical chest pain who are evaluated for acute aortic syndrome (AAS) or pulmonary embolism (PE) with computed tomoangiography (CTA) and discuss the rationale for the use of triple rule-out (TRO) protocol for triaging these patients.
\end{abstract}

Methods: This study is a retrospective analysis of patients presenting with atypical chest pain and evaluated with thoracic (CTA), for suspicion of AAS/PE. Two physicians reviewed patient files for demographic characteristics, initial CT and final clinical diagnosis. Patients were classified according to CTA finding into AAS, PE and other diagnoses and according to final clinical diagnosis into AAS, PE, ACS and other diagnoses.

Results: Four hundred and sixty-seven patients were evaluated: 396 (84.8\%) patients for clinical suspicion of PE and 71 (15.2\%) patients for suspicion of AAS. The prevalence of ACS and AAS was low among the PE patients: $5.5 \%$ and $0.5 \%$ respectively $(\mathrm{P}=0.0001)$, while the prevalence of ACS and PE was $18.3 \%$ and 5.6\% among AAS patients $(\mathrm{P}=0.14$ and $\mathrm{P}=0.34$ respectively).

Conclusion: The prevalence of ACS and AAS among patients suspected clinically of having PE is limited while the prevalence of ACS and $\mathrm{PE}$ among patients suspected clinically of having AAS is significant. Accordingly patients suspected for PE could be evaluated with dedicated PE CTA while those suspected for AAS should still be triaged using TRO protocol.

Manuscript accepted for publication May 08, 2015

aDepartment of Radiology \& Medical Imaging, Armed Forces Hospital, Southern Region, Saudi Arabia

${ }^{b}$ Cardio-Thoracic and Vascular Unit, Department of Radiology, University Hospital of Lausanne, Switzerland

${ }^{c}$ Quantitative Medical Imaging Laboratory, Department of Radiology, University Hospital of Lausanne, Switzerland

${ }^{\mathrm{d} C o r r e s p o n d i n g ~ A u t h o r: ~ S a a d ~ A l ~ Q a h t a n i, ~ D e p a r t m e n t ~ o f ~ R a d i o l o g y ~ \& ~ M e d i-~}$ cal Imaging, Armed Forces Hospital, Southern Region, Khamis Mushayt 61961, PO Box 101, Saudi Arabia. Email: dr.salqahtani@gmail.com

doi: http://dx.doi.org/10.14740/jocmr2197w
Keywords: Acute coronary syndrome; Acute aortic syndrome; Pulmonary embolism; Triple rule-out

\section{Introduction}

Patients who present to the emergency department (ED) with chest pain constitute a common and important diagnostic challenge. In the Centers for Disease Control and Prevention survey, evaluation of acute chest pain and related symptoms was the second most common reason for a visit to the ED by a female adult and the most common reason by a male adult in the United States [1-3]. Chest pain accounted for more than 8 million of the ED visits $[2,3]$ and for about 2 million hospital admissions per year [4].

Among the most clinically relevant conditions causing chest pain that have to be differentiated in the ED are pulmonary embolism (PE), acute aortic syndromes (AAS), and acute coronary syndrome (ACS). The last condition is identified in approximately $15-25 \%$ of patients with acute chest pain who are evaluated in EDs [5]. Unfortunately, the number of patients with manifestations of acute myocardial infarction who are inappropriately discharged from the ED is not negligible, about $2-5 \%$ of patients $[6,7]$.

Patients in whom the diagnosis of ACS is missed tend to be younger and to have an atypical presentation and a nondiagnostic electrocardiogram (ECG) [8]. The missed diagnosis of ACS is a common reason for litigation against ED physicians and accounts for up to $25 \%$ of the total malpractice liability of ED physicians [9]. On the other hand, uncertainty in the diagnosis of ACS results in the practice of defensive medicine and begets an increased number of diagnostic tests and hospital admissions [10]. The cost of negative inpatient cardiac evaluations is estimated at \$6 billion in the United States each year [11].

The one-step CT examination for chest pain, the so-called "triple rule-out (TRO) protocol" used to diagnose ACS, PE, and AAS is increasingly being performed in many institutions equipped with 64-slice or higher multi-detector CT scanners. Although there have been many articles dealing with TRO, these studies did not provide detailed data of delayed diagnosis in assessing patients with atypical chest pain who do not undergo immediate cardiac computed tomoangiography (CTA) 
Table 1. Incidence of PE, AAS and ACS Among Patients With Initial Clinical Suspicion of PE or AAS

\begin{tabular}{lllll|}
\hline \multirow{2}{*}{ Pre-test diagnosis } & \multicolumn{4}{c|}{ Post-test diagnosis } \\
\cline { 2 - 5 } & PE & AAS & ACS & Others \\
\hline PE $(\mathrm{n}=396)$ & $91(23 \%)$ & $2(0.5 \%)$ & $22(5.5 \%)$ & $281(71 \%)$ \\
AAS $(\mathrm{n}=71)$ & $4(5.6 \%)$ & $7(9.9 \%)$ & $13(18.3 \%)$ & $47(66.2 \%)$ \\
Total $(\mathrm{n}=467)$ & $95(20.3 \%)$ & $9(2 \%)$ & $35(7.5 \%)$ & $328(70.2 \%)$ \\
\hline
\end{tabular}

[4, 11-19]. In addition, there is limited information regarding overall concepts, such as how to choose the optimal examination (i.e., dedicated coronary CTA versus TRO versus dedicated PE or aortic CTA) based on the specific clinical presentation to the ED [4]. Moreover, the actual incidence of ACS, PE and AAS and the overlap between the three entities in patients presenting to ED with atypical chest pain remains unclear. There are no enough studies describing the overlap between the ACS from a side and the PE/AAS from another side.

The aims of this study were to evaluate the prevalence of ACS among patients with atypical chest pain who are evaluated for AAS or PE with CTA and accordingly discuss the rationale for the use of TRO protocol for triaging these patients.

\section{Materials and Methods}

The study consisted of retrospective review of all consecutive outpatients who presented with atypical chest pain and underwent thoracic CTA, for clinical suspicion of PE and/or AAS, during a consecutive 12 months period at our University Hospital. The hospital's institutional review board and ethical committee approved the study.

\section{Eligibility criteria}

All patients 18 years or older who presented with a clinical history of non-traumatic atypical or non-angina acute chest pain, suggestive of AAS or PE and who underwent thoracic CTA were included. Definition of atypical or non-angina chest pain was based on the guidelines recently reported by the National Institute of Clinical Excellence (NICE) [20]. Typical angina combines the three following criteria: 1) constricting discomfort in the front of the chest or the neck, shoulders, jaw or arms, 2 ) precipitated by physical exercise and 3) relieved by rest or glyceryl trinitrate (GTN) within about $5 \mathrm{~min}$. Atypical chest pain is defined as pain with two of the three criteria and nonangina pain with one or none of the three criteria.

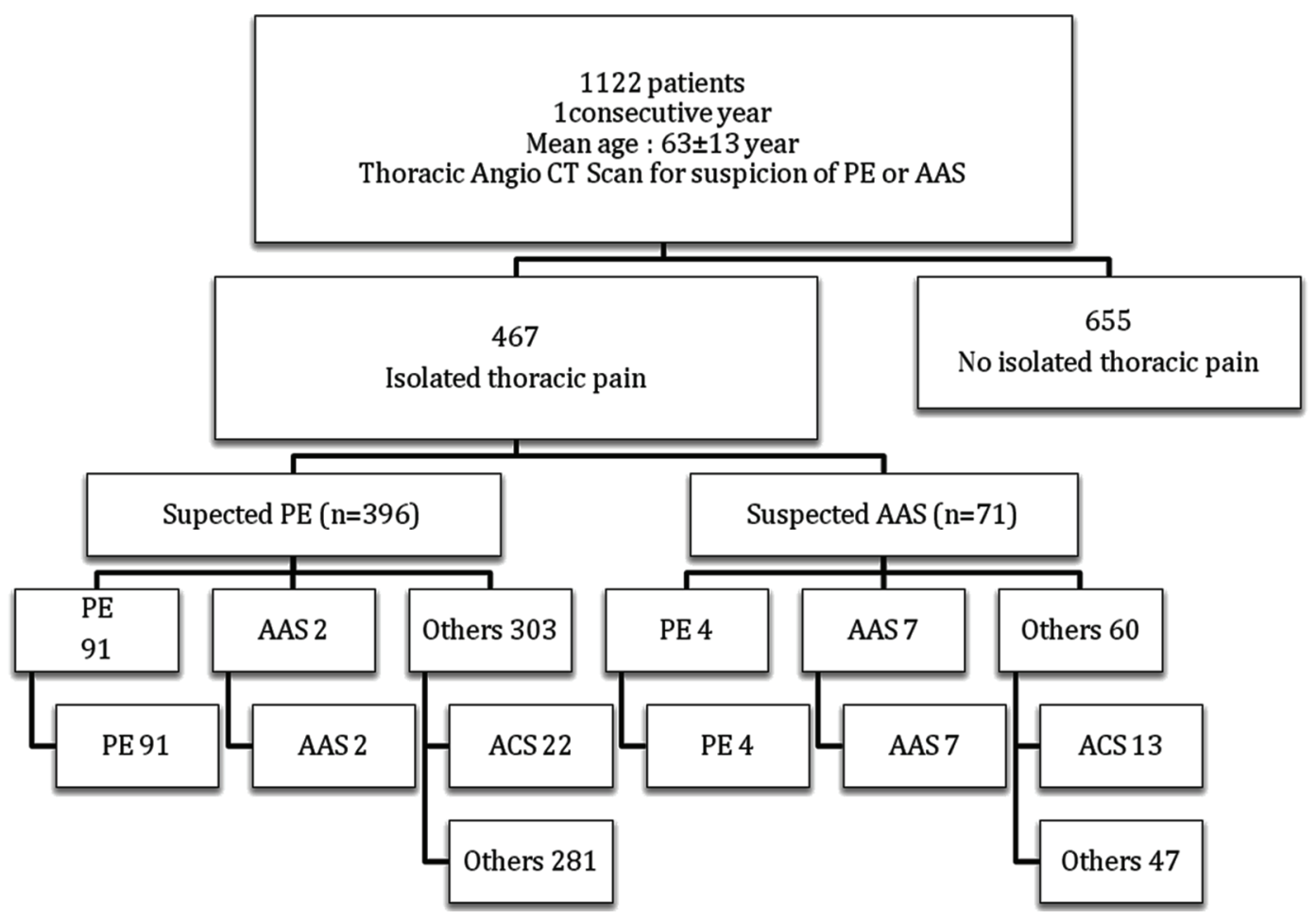

Figure 1. Final diagnosis among 467 patients with atypical chest pain evaluated with CTA. 
Table 2. Overlap Between PE, ACS and AAS Among Patients With Initial Clinical Suspicion of PE

\begin{tabular}{lllll}
\hline & Prevalence of PE & Prevalence of ACS & Prevalence of AAS & P \\
\hline Pre-PE $(\mathrm{N}=396)$ & $23 \%$ & $5.5 \%$ & & 0.0001 \\
& $23 \%$ & & $0.5 \%$ & 0.0001 \\
\hline
\end{tabular}

Clinical features suggestive of AAS included chest pain, nausea, and diaphoresis, extreme apprehension with a sense of impending doom, associated focal neurologic symptoms or peripheral arterial ischemia. Clinical features suggestive of PE included chest pain, associated shortness of breath, and dizziness. We used the pre-test score to stratify patients on low, intermediate and high probability of PE. Patients with low/ intermediate probability and positive D-dimer test as well as patients at high probability were indication for thoracic CTA [21].

\section{Exclusion criteria}

By design, patients without chest pain and those with clinical ECG (i.e. ST elevation or depression of more than $1 \mathrm{~mm}$ or T-wave inversion in more than two anatomically continuous leads) or laboratory evidence (elevation of cardiac biomarkers) suggestive of ACS and patients with history of ACS were also excluded from the study.

\section{Data analysis}

Two physicians reviewed in consensus the patients' files for demographic characteristics, initial or suspected clinical diagnosis (pre-test diagnosis), CT diagnosis and the final diagnosis (post-test diagnosis). The final diagnosis was established using the discharge diagnosis and/or 30 days patient outcome.

Patients were then classified according to their CT finding into AAS, PE or other diagnoses and according to their final diagnosis as having AAS, PE, ACS or other diagnoses.

Quantitative variables were expressed as means \pm standard deviations and categorical variables percentages. We calculated the percentage of patients who had a final diagnosis of either PE or AAS (post-test diagnosis), among the patients with an initial suspicion of PE or AAS (pre-test diagnosis). The percentage of patients with a final diagnosis of ACS in each of the previous two groups, PE or AAS, was also calculated as well as among the whole population with atypical chest pain.

The Pearson's Chi-square $\left(\mathrm{X}^{2}\right)$ test was used to evaluate whether the results were statistically significant or not. A P value $<0.05$ was considered significant.

\section{Results}

Four hundred and sixty-seven (467) patients (249 male and 218 female) presenting with atypical chest pain met the eligibility criteria for inclusion in the study. They included 396 (84.8\%) patients (mean age 62.3 years) with initial clinical suspicion of having PE (pre-test PE group) and 71 (15.2\%) patients (mean age 58.1 years) initially suspected of having AAS (pre-test AAS group).

The final diagnosis (post-test) of the 467 patients in the study included 95 patients with PE, nine patients with AAS, 35 patients with ACS and 328 patients with other diagnosis. The initial clinical suspicion of the 95 patients with final diagnosis of PE included 91 patients $(96 \%)$ with PE (pre-test PE) and four patients (4\%) with AAS (pre-test AAS) while the initial clinical suspicion of the nine patients with AAS included seven patients $(77 \%$ ) with AAS (pre-test AAS) and two patients (22\%) with PE (pre-test PE) (Table 1).

The final diagnosis of the 396 patients, initially suspected of having PE, included $91(23 \%)$ patients with PE, two $(0.5 \%)$ patients with AAS, $22(5.5 \%)$ patients with ACS and $281(71 \%)$ patients with other diagnoses. On the other hand, among the 71 patients initially suspected of having AAS, the final diagnosis involved seven $(9.9 \%)$ patients with AAS, four (5.6\%) patients with PE, $13(18.3 \%)$ patients with ACS and 47 (66.2\%) patients with other diagnoses (Fig. 1, Table 1).

Thirty-five patients had a final diagnosis of ACS (22 in the pre-test PE group and 13 in the pre-test AAS group), accounting for a $7.5 \%$ of all patients presenting with atypical chest pain in the study (Table 1).

The prevalence of ACS and AAS was low in the pre-test PE group, seen in only $5.5 \%$ and $0.5 \%$ of the patients of that group respectively $(\mathrm{P}=0.0001)$ (Table 2$)$. On the other hand, the prevalence of ACS was higher $(18.3 \%)$ than the prevalence of AAS $(9.9 \%)$ in the pre-test AAS group $(\mathrm{P}=0.14)$. In this group of patients, the prevalence of PE $(5.6 \%)$ was lower than AAS $(9.9 \%)(\mathrm{P}=0.34)$ (Table 3$)$.

\section{Discussion}

CTA has become a standard procedure in the evaluation of PE and AAS [22]. Cardiac CTA has proved to be an effective tool to rule out coronary artery disease (CAD), with a sensitivity of

Table 3. Overlap Between PE, ACS and AAS Among Patients With Initial Clinical Suspicion of AAS

\begin{tabular}{lllll}
\hline & Prevalence of AAS & Prevalence of ACS & Prevalence of PE & P \\
\hline Pre-AAS $(\mathrm{N}=71)$ & $9.9 \%$ & $18.3 \%$ & & 0.14 \\
& $9.9 \%$ & $5.6 \%$ & 0.34 \\
\hline
\end{tabular}


93-99\% and negative predictive value of 95-99\% [23]. About $20 \%$ of patients with acute chest pain present to the ED with atypical acute chest pain and often require multiple examinations to exclude PE and/or AAS, in addition to excluding obstructive CAD [24].

It has been suggested that despite its high negative predictive value for $\mathrm{CAD}$, cardiac $\mathrm{CT}$ has a limited capacity to diagnose non-cardiac causes of chest pain arising from sources outside the anatomic window it interrogates. A more comprehensive evaluation that rapidly excludes both cardiac and noncardiac sources of chest pain should theoretically be preferable. Technical advances in cardiac CT and intravenous contrast injection protocols have made a TRO protocol feasible, which can effectively image the coronary, aortic, and pulmonary arterial beds $[4,6,15-18]$. The TRO protocol allows for simultaneous evaluation and exclusion of $\mathrm{CAD}, \mathrm{PE}$, and AAS in a single rapid test.

By potentially eliminating the need for further diagnostic testing, a TRO approach seems to improve the efficiency and downstream clinical outcomes of acute chest pain evaluation [25]. However, the implementation of TRO protocol is not without some drawbacks. Compared with standard cardiac CTA, TRO approach is associated with high radiation exposure longer breath-hold and increased contrast volume. The additional radiation exposure is attributable to the greater scan length required to image the entire pulmonary arterial circulation and thoracic aorta. In a comparative study between TRO protocol and standard CTA, it was reported that TRO protocol was associated with $46 \%$ higher radiation exposure than standard CTA [26]. Two studies showed that TRO protocol did not show any improvement of diagnostic yield, regarding ACS, PE or AAS, clinical outcomes (90 days outcome), cost of care or downstream health care resources use as compared with standard CTA $[22,25]$.

Another very interesting issue that should be considered when considering the implementation of TRO protocol is the incidence of ACS, PE and AAS and the overlap between the three entities in patients presenting to the ED with atypical chest pain. To our knowledge, this is the first study to show the prevalence and overlap of these three entities in a large cohort of patients. In this study, the final diagnostic yield for ACS, PE or AAS was $7.5 \%, 20.3 \%$ and $2 \%$ respectively. In a recent study evaluating the diagnostic yield of TRO protocol among 272 patients with acute chest pain, the prevalence of ACS, PE and AAS was $13.2 \%, 1.1 \%$ and $0 \%$ respectively [25]. According to the same study, the low diagnostic yield of TRO protocol for PE and AAS could be attributed to the inappropriate patient selection, including indiscriminate TRO use in patients with low pre-test probability which seemed to be driven by the clinical implications of death, disability, and litigation resulting from missed diagnosis in these cases [25].

The results of our study clearly indicate that within the pre-test PE patients, the overlap between PE $(23 \%)$ on one hand and either ACS (5.5\%) or AAS (0.5\%) on the other hand is limited. Similarly in a small cohort of patients with high pretest probability of PE, one study demonstrated a $21 \%$ PE yield using TRO protocol [15]. This study also demonstrated a poor overlap between PE on one hand and AAS (4\%) or ACS (3\%) on the other hand in their patients.

On the other hand, our study demonstrated a greater overlap between AAS and either ACS or PE among the pre-test AAS patients with the overlap being higher between AAS and ACS. This is in agreement with Yoo et al [2] who stated that AAS is much less frequent compared with ACS and there is substantial overlap in clinical symptoms and signs between ACS and AAS, ED physicians tend to mistake AAS for ACS, and the reverse also occurs. Furthermore, type A aortic dissection could be associated with coronary artery flow impairment that could mimic ACS clinical presentation.

Therefore we believe that our results are strong enough to suggest that patients presenting to ED with atypical chest pain with pre-test probability of PE should be considered for a dedicated CTA for PE only. On the other hand, those patients with a pre-test probability of AAS should be examined with either TRO protocol or a dedicated coronary CTA with extended volume of interest in the $\mathrm{z}$ direction to cover the entire thoracic aorta (double-rule-out protocol). A possible advantage of such latter approach is to better analyze the ascending aorta and the potential extension of the aortic dissection to the coronary arteries with ECG gated protocols.

The previously proposed algorithm might help to minimize the number of TRO protocol being performed today in favor of dedicated coronary or PE CTA. This will minimize the dose of radiation exposure and volume of contrast given to those patients as well as reduce the number of studies requiring interpretation by radiologists with special CTA experience.

Meanwhile, it also seems logical at the time being to highlight the importance of applying strict clinical criteria for patient selection rather than performing systematic TRO protocol when investigating patients with acute chest pain.

Further larger prospective randomized trials comparing these two techniques for patients presenting to ED with clinical suspicion of AAS might help to sort out this issue.

\section{Conclusion}

The overlap between PE, AAS and ACS among patients with atypical chest pain suspected clinically of having PE is limited while the overlap between the three conditions among patients with clinical suspicion of AAS is greater and significant. Therefore, we believe that patients presenting to ED with pre-test probability of PE should be triaged for dedicated PE CTA while those with pre-test probability of AAS should be still triaged for a TRO protocol or modified cardiac CTA. The application of strict clinical criteria for the application of TRO protocol in patients with atypical chest pain is recommended.

\section{Abbreviations}

AAS: acute aortic syndrome; ACS: acute coronary syndrome; PE: pulmonary embolism; TRO: triple rule-out; ED: emergency department; MDCT: multi-detector computed tomography; ECG: electrocardiogram; CTA: computed tomoangiography 


\section{References}

1. Pitts SR, Niska RW, Xu J, Burt CW. National Hospital Ambulatory Medical Care Survey: 2006 emergency department summary. Natl Health Stat Report. 2008;(7):138.

2. Shreibati JB, Baker LC, Hlatky MA. Association of coronary $\mathrm{CT}$ angiography or stress testing with subsequent utilization and spending among Medicare beneficiaries. JAMA. 2011;306(19):2128-2136.

3. Amsterdam EA, Kirk JD, Bluemke DA, Diercks D, Farkouh ME, Garvey JL, Kontos MC, et al. Testing of lowrisk patients presenting to the emergency department with chest pain: a scientific statement from the American Heart Association. Circulation. 2010;122(17):1756-1776.

4. Yoo SM, Rho JY, Lee HY, Song IS, Moon JY, White CS. Current Concepts in Cardiac CT Angiography for Patients With Acute Chest Pain. Korean Circ J. 2010;40(11):543549.

5. Pope JH, Selker HP. Acute coronary syndromes in the emergency department: diagnostic characteristics, tests, and challenges. Cardiol Clin. 2005;23(4):423-451, v-vi.

6. Pope JH, Aufderheide TP, Ruthazer R, Woolard RH, Feldman JA, Beshansky JR, Griffith JL, et al. Missed diagnoses of acute cardiac ischemia in the emergency department. N Engl J Med. 2000;342(16):1163-1170.

7. Rusnak RA, Stair TO, Hansen K, Fastow JS. Litigation against the emergency physician: common features in cases of missed myocardial infarction. Ann Emerg Med. 1989;18(10):1029-1034.

8. Karcz A, Korn R, Burke MC, Caggiano R, Doyle MJ, Erdos MJ, Green ED, et al. Malpractice claims against emergency physicians in Massachusetts: 1975-1993. Am J Emerg Med. 1996;14(4):341-345.

9. Katz DA, Williams GC, Brown RL, Aufderheide TP, Bogner M, Rahko PS, Selker HP. Emergency physicians' fear of malpractice in evaluating patients with possible acute cardiac ischemia. Ann Emerg Med. 2005;46(6):525-533.

10. Storrow AB, Gibler WB. Chest pain centers: diagnosis of acute coronary syndromes. Ann Emerg Med. 2000;35(5):449-461.

11. Raff GL, Gallagher MJ, O'Neill WW, Goldstein JA. Diagnostic accuracy of noninvasive coronary angiography using 64-slice spiral computed tomography. J Am Coll Cardiol. 2005;46(3):552-557.

12. Manghat NE, Morgan-Hughes GJ, Roobottom CA. Multi-detector row computed tomography: imaging in acute aortic syndrome. Clin Radiol. 2005;60(12):1256-1267.

13. Rathbun SW, Raskob GE, Whitsett TL. Sensitivity and specificity of helical computed tomography in the diagnosis of pulmonary embolism: a systematic review. Ann Intern Med. 2000;132(3):227-232.

14. Hiorns MP, Mayo JR. Spiral computed tomography for acute pulmonary embolism. Can Assoc Radiol J. 2002;53(5):258-268.

15. Schertler T, Frauenfelder T, Stolzmann P, Scheffel H, Des- biolles L, Marincek B, Kaplan V, et al. Triple rule-out CT in patients with suspicion of acute pulmonary embolism: findings and accuracy. Acad Radiol. 2009;16(6):708-717.

16. Johnson TR, Nikolaou K, Wintersperger BJ, Knez A, Boekstegers P, Reiser MF, Becker CR. ECG-gated 64MDCT angiography in the differential diagnosis of acute chest pain. AJR Am J Roentgenol. 2007;188(1):76-82.

17. Vrachliotis TG, Bis KG, Haidary A, Kosuri R, Balasubramaniam M, Gallagher M, Raff G, et al. Atypical chest pain: coronary, aortic, and pulmonary vasculature enhancement at biphasic single-injection 64-section CT angiography. Radiology. 2007;243(2):368-376.

18. Lee HY, Yoo SM, White CS. Coronary CT angiography in emergency department patients with acute chest pain: triple rule-out protocol versus dedicated coronary CT angiography. Int J Cardiovasc Imaging. 2009;25(3):319326.

19. Hirai N, Horiguchi J, Fujioka C, Kiguchi M, Yamamoto H, Matsuura N, Kitagawa T, et al. Prospective versus retrospective ECG-gated 64-detector coronary CT angiography: assessment of image quality, stenosis, and radiation dose. Radiology. 2008;248(2):424-430.

20. Anon CG95. Chest pain of recent onset: NICE guideline. NICE. 2010.

21. Hedner C, Sundgren PC, Kelly AM. Associations between presence of relevant information in referrals to radiology and prevalence rates in patients with suspected pulmonary embolism. Acad Radiol. 2013;20(9):1115-1121.

22. Rogers IS, Banerji D, Siegel EL, Truong QA, Ghoshhajra BB, Irlbeck T, Abbara S, et al. Usefulness of comprehensive cardiothoracic computed tomography in the evaluation of acute undifferentiated chest discomfort in the emergency department (CAPTURE). Am J Cardiol. 2011;107(5):643-650.

23. Hoffmann U, Moselewski F, Cury RC, Ferencik M, Jang IK, Diaz LJ, Abbara S, et al. Predictive value of 16-slice multidetector spiral computed tomography to detect significant obstructive coronary artery disease in patients at high risk for coronary artery disease: patient-versus segmentbased analysis. Circulation. 2004;110(17):2638-2643.

24. Rogg JG, De Neve JW, Huang C, Brown D, Jang IK, Chang Y, Marill K, et al. The triple work-up for emergency department patients with acute chest pain: how often does it occur? J Emerg Med. 2011;40(2):128-134.

25. Madder RD, Raff GL, Hickman L, Foster NJ, McMurray MD, Carlyle LM, Boura JA, et al. Comparative diagnostic yield and 3-month outcomes of "triple rule-out" and standard protocol coronary CT angiography in the evaluation of acute chest pain. J Cardiovasc Comput Tomogr. 2011;5(3):165-171.

26. Takakuwa KM, Halpern EJ, Gingold EL, Levin DC, Shofer FS. Radiation dose in a "triple rule-out" coronary CT angiography protocol of emergency department patients using 64-MDCT: the impact of ECG-based tube current modulation on age, sex, and body mass index. AJR Am J Roentgenol. 2009;192(4):866-872. 